\title{
When hematochezia becomes a red herring
}

\author{
S. Smeets ${ }^{1}$, F. Dedeurwaerdere ${ }^{2}$, E. Thomaere ${ }^{3}$, B. Houthoofd ${ }^{3}$, L. D’Hulst ${ }^{4}$, P. Wilmes ${ }^{5}$, F. Offner ${ }^{6}$, S. De Coninck ${ }^{7}$
}

(1) Gastroenterologist in training, University hospital of Ghent; (2) Department of anatomopathology, Sint-Andries hospital, Tielt; (3) Department of radiology, Sint-Andries hospital, Tielt; (4) Department of nulcear medicine, Sint-Andries hospital, Tielt; (5) ; Department of oncology, Sint-Andries hospital, Tielt; (6) Department of hematology, University hospital, Ghent; (7) Department of gastroenterology, Sint-Andries hospital, Tielt.

\section{Question}

An 81-year-old male patient presented on the emergency ward for hematochezia, without any other complaints. Rectosigmoidoscopy showed a predominant patchy inflammation of the mucosa with several bluish blebs (submucosal hemorrhage) and small ulcerations. (Figure 1A). Subsequent elective colonoscopy (1) revealed multiple submucosal hematomas and mucosal lacerations throughout the entire colon in between normal mucosa. During the procedure spontaneous mucosal tears occurred. (Figure 1B). Multiple biopsies were taken.

What is the diagnosis?

\section{Answer}

Pathology revealed submucosal amyloid deposition (with Congo-red staining). (Figure 2A) Immunohistochemistry was positive for lambda light chains and negative for amyloid AA. Transthyretin was also positive. (Figure 2B). All suggestive for a senile amyloidosis.

Further investigations excluded systemic and cardiac amyloidosis. Biopsies in the esophagus, stomach or duodenum were normal. The diagnosis of an isolated senile amyloidosis (ATTRwt) of the colon was made.

Due to a variety and non-specificity of symptoms (weight loss, diarrhea, abdominal pain, malabsorption, esophageal reflux, varying degrees of upper and lower GI bleeding), diagnosis of gastro-intestinal amyloidosis is often delayed (2). The gold standard is Congo-red staining on a tissue sample of the affected organ (3).

Treatment of acquired amyloidosis is treating the possible underlying disorder (malignancy, infection, auto-immune disorder), together with treating the symptoms.
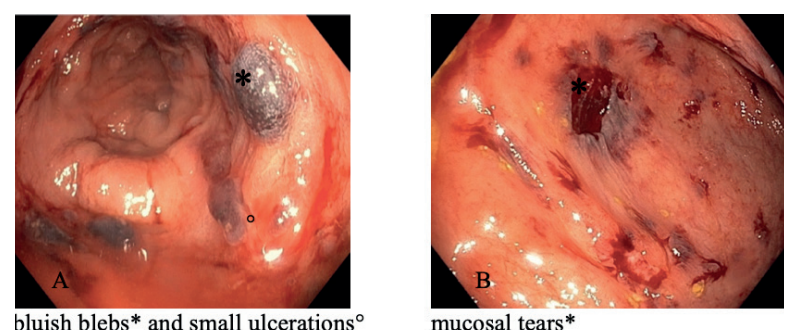

Figure 1.

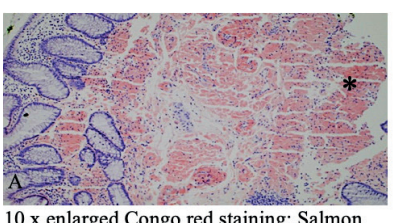

$10 \mathrm{x}$ enlarged Congo red staining: Salmon pink coloring of amyloid*

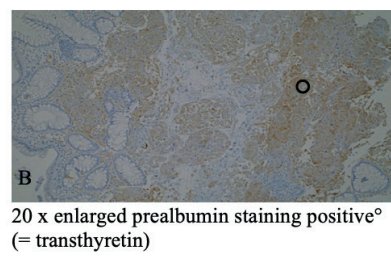

Figure 2.
For ATTRwt isolated to the colon we could not found a therapeutic strategy in the literature. Our patient was treated with beclomethasone $5 \mathrm{mg}$ once daily, with remission of blood loss and complete endoscopic healing. However, to date no repeat biopsies were taken.

As far as we know, this is the first case of a symptomatic isolated ATTRwt amyloidosis of the colon.

\section{References}

1. MOSLI M, ALDABBAGH A, ASEERI H, et al. The diagnostic yield of urgent colonoscopy in acute lower gastrointestinal bleeding. Acta Gastroenterol Belg. 2020 Apr-Jun; 83(2): 265-270.

2. EBERT E., NAGARM. Gastrointestinal Manifestations of Amyloidosis. Am J Gastroenterol., 2008; 103: 776-787.

3. WECHALEKAR A., GILLMORE J., HAWKINS P. Systemic amyloidosis. Lancet, 2015; 387: 2641-2654. 